\title{
El tratamiento completo: alternativa para la traducción del verso inserto en prosa no literaria
}

\author{
Bianchinetta Benavides Segura \\ Universidad Nacional, Costa Rica
}

\section{Resumen}

Se describen aspectos teóricos y la aplicación del denominado tratamiento completo, en la traducción de fragmentos poéticos. Se examinan los potenciales efectos en el texto terminal, a partir de un cuidadoso análisis del original; por tanto, el componente cultural cobra importancia y valor metodológicos. Se profundiza en los rasgos particulares del discurso poético como género, desde los cuales se extraen propuestas de trabajo (doce pasos) y varias soluciones traductológicas.

\begin{abstract}
A description is provided of the theoretical framework and application of the so-called complete treatment, in the translation of poetic fragments. Their potential effects on the target text are examined, based on a careful analysis of the source text, thus giving the cultural component methodological importance and value. Specific aspects of poetry as a genre are discussed in depth, as a basis for the proposal of twelve steps to follow and for different solutions for translation problems.
\end{abstract}

El presente artículo expone los fundamentos teóricos utilizados para la creación de la metodología denominada el tratamiento completo, la cual surge del proceso de traducción aplicado a los fragmentos 
de poemas insertos dentro de la prosa no literaria del libro North Carolina and Old Salem Cookery ${ }^{1}$. Posterior a la traducción de la obra, se elaboró un análisis del procedimiento traductológico seguido con seis fragmentos de poesía de una gran cantidad literaria, cuya función es la de conectar al lector del texto original (T.O.) con una gran cantidad de elementos extratextuales transmisores de sentido subyacente.

Lejos de ser un simple compendio de recetas, North Carolina and Old Salem Cookery es más bien una descripción del estilo de vida predominante en Carolina del Norte durante los años previos y posteriores a la depresión económica causada por la Guerra Civil. El texto completo se divide en dos partes: una narración de las vivencias de la autora relacionadas con la cultura culinaria de su tierra natal y un compendio de recetas de cocina, en el que se incluyen platillos típicos tradicionales de la región sur de los Estados Unidos. Este libro fue escrito para hacer conciencia acerca de la importancia de rescatar la riqueza culinaria local y ofrecerles a las futuras generaciones un documento con instrucciones para preparar aquellos platillos que padres y abuelos conocieron durante la niñez.

En un libro de cocina no se esperaría encontrar tantas consideraciones referentes a la cultura, tradiciones y vivencias personales de su autora, muchas de las cuales bien pudieron pasarse por alto al trasladarlo al castellano, de no haberse considerado su importancia para los propósitos de la obra. Al igual que en el caso de la traducción de poemas completos, la traducción de fragmentos poéticos insertos apunta al rescate de la forma, el tono y el estilo como creadores de sentido. En la traducción de la prosa no literaria se impone la denotación: en las secciones correspondientes a las recetas de cocina, se debe velar por la reproducción en extremo cuidadosa de cada ingrediente, cada medida, cada indicación, con el fin de lograr un sabroso resultado. La traducción del texto poético inserto debe apuntar hacia la

1. Beth Tartan, North Carolina and Old Salem Cookery (Chapel Hill: The University of North Carolina Press, 1992). 
connotación: esos elementos que transportan al lector terminal a redescubrir los propios recuerdos de antaño capaces de producir un efecto con mucho más sabor.

Los procedimientos utilizados en la primera etapa de la traducción de esos fragmentos incluyeron la revisión de los conceptos teóricos existentes con respecto a la traducción del género literario ${ }^{2}$. Sobre la traducción de poesía, existen dos posiciones contrarias. La primera la presenta como un ente intraducible; hay quienes se oponen por completo a la traducción del verso e inclusive condenan el rescate del ritmo o la rima. Esta posición, bastante compartida entre los teóricos no contemporáneos, prefiere reproducir el contenido del texto poético con absoluta exactitud haciendo uso de recursos ajenos a la forma y al estilo poético, tales como la prosa explicativa o la presencia de extensas notas al pie de página, cuyo objetivo es situar al lector de la traducción en el contexto subyacente en el poema. Este tipo de metodología resulta más bien apropiado para libros de texto, resúmenes de crítica y análisis literario, pero no para el rescate de la forma artística.

El presente análisis se fundamenta en teóricos más recientes, quienes plantean una metodología de traducción distinta en donde se asume que, si el autor del T.O. le presenta a su lector textos poéticos naturalmente conformados de estilo, tono, forma y contenido semántico, el traductor habrá de desarrollar las estrategias y la metodología específicas para la creación de una versión que funcione de la misma manera. Esta metodología se centró en el desglose y planteamiento de la traducción de los fragmentos de verso popular insertos en el T.O., los cuales provienen de la poesía popular de tema folclórico, es decir, coplas, poemas y canciones populares, y de la poesía popular de tema religioso: plegarias de acción de gracias y villancicos. Estas muestras de verso inserto en la prosa evocan la identidad cultural; en sí mismas, constituyen una antología de tradiciones.

2. Dada la riqueza del concepto "poesía", el corpus de este análisis se limita al subgénero de poesía popular. 


\section{Consideraciones teóricas sobre la traducción de los fragmentos poéticos insertos}

Lejos de limitarse a enumerar ingredientes o a presentar simples métodos de preparación de alimentos, North Carolina and Old Salem Cookery evoca una época de conocimiento y habilidades culinarias, estableciendo así un vínculo con un pasado y estilo de vida específicos. Cada grupo de recetas viene precedido de las anécdotas, refranes, canciones y vivencias de quienes las disfrutaron a principios del siglo xx. Como resultado, el lector original percibe la influencia de dicho pasado en su propia identidad actual.

La propuesta aquí descrita pretende que el lector terminal también experimente sentimientos emotivos que le permitan revivir el sabor de su propio pasado, y esto implica analizar y reevaluar las distintas estrategias traductológicas que apunten al rescate de la "función apelativa" 3 de ambos subgéneros literarios: el de la prosa no literaria y el de la poesía popular. En la primera etapa se consideró el grado de literalidad o de cambio en la forma de los fragmentos, siempre en función del rescate de lo apelativo. La forma de los versos no se apega, entonces, a la estructura denotativa, dado que "el traductor que se contenta con los modismos del original, o no los diferencia o no los matiza, da impresión de pobreza... El traductor que traduzca los modismos sin correspondencia analítica, palabra por palabra, sacará un sentido que deformará el contenido del original" ${ }^{\circ 4}$.

La forma en que encontramos los fragmentos presume un interés por dirigir la atención del lector en una dirección semántica distinta a la de información en las otras secciones. Con respecto al valor semántico de la poesía y su influencia en el lector, H.G. Widdowson ${ }^{5}$ indica que:

3. Eugene Nida, La traducción: teoría y práctica (Madrid: Ediciones Cristiandad, 1986) 163.

4. Luis Alonso Schökel y Eduardo Zurro, La traducción bíblica: lingüística y estilística (Madrid: Ed. Cristiandad, 1977) 156.

5. H.G. Widdowson, Practical Stylistics: An Approach to Poetry (Nueva York: Oxford University Press, 1992) 11. 
...they [poems] are meant to be interpreted as having some relevance to human affairs. Their very appearance in print implies they have something of significance to say. Although they may express an individual, even idiosyncratic, perception, they are not private but public statements and so make some claim to be relevant to other people's lives.

Muchas de las soluciones traductológicas que se presentan son efectivamente idiosincráticas dadas las múltiples semejanzas entre las culturas culinarias y de tradiciones de la vida hogareña de los lectores original y terminal. Resulta prudente preguntarse si las mismas resultan apropiadas en la búsqueda de la fidelidad semántica de la traducción de los fragmentos poéticos. Para este cuestionamiento, encontramos varias respuestas. Hatim y Mason ${ }^{6}$ afirman que "las motivaciones del traductor están inseparablemente ligadas al contexto sociocultural en el que se produce el acto de traducir". Más adelante agregan que:

Es sólo cuando el traductor se propone a dar cuenta del "espíritu" y no del mero sentido del texto cuando comienza en realidad a traducir... en lugar de aplicar algún criterio nocional que sirva para calificar de buena una traducción de poesía, las versiones pueden ser juzgadas con arreglo a lo que el traductor se ha propuesto alcanzar en cada caso ${ }^{7}$.

Christiane Nord ${ }^{8}$ explica que, dada su función de iniciador del texto terminal (T.T.), el traductor está obligado a analizar el papel que su contexto sociocultural juega en la transmisión del mensaje:

6. Basil Hatim e Ian Mason, Teoría de la traducción: una aproximación al discurso (Barcelona: Editorial Ariel, 1995) 23.

7. Hatim y Mason, 26-27.

8. Christiane Nord, Text Analysis in Translation (Amsterdam: Editions Rodopi BV, 1991) 21. 
By means of a comprehensive model of text analysis, which takes into account intratextual as well as extratextual factors, the translator can establish the "function in culture" of a source text. He then compares this with the prospective function-inculture of the target text required by the initiator, identifying and isolating those source text elements which have to be preserved or adapted in translation.

Yves Bonnefoy ${ }^{9}$ amplía la importancia de analizar el contexto de la poesía a partir de las vivencias del traductor, quien ahora se constituye en un nuevo poeta: “...you must live before you write... the poem is nothing and that translation is possible-which is not to say that it is easy; it is merely poetry re-begun".

Nuestra propuesta será, entonces, "una recodificación interpretativa en donde únicamente cabe la transposición creadora de una forma poética a otra forma poética"10 y uno de los fundamentos del método propuesto es el criterio de que la traducción de los fragmentos poéticos habrá de considerarse como sinónimo de cambio. Peter Newmark" indica que "The translator's task is never to copy what is said, but to put himself in the direction of what is said in order to carry over what is to be said". En la búsqueda de la recodificación que coloque al lector terminal en dirección de lo que se ha dicho en el T.O., se ha intentado crear una forma poética natural. La naturalidad misma es un concepto "easy to define but not easy to concrete about... Naturalness acquired by instincts depends on the relationship between the writer and the readership" ${ }^{\prime 2}$. Esta naturalidad está impregnada del contexto folclórico de ambas culturas, y dado que el folclore es “...a traditional, unofficial, non-institutional part of culture, it

9. Yves Bonnefoy, The Act and the Place of Poetry: Selected Essays (Chicago: The University of Chicago Press, 1989) 189.

10. Roman Jakobson, "En torno a los aspectos lingüísticos de la traducción". Ensayos de lingüística general (Barcelona: Seix-Barral, 1975) 66-77.

11. Peter Newmark, A Textbook of Translation (Nueva York: Prentice Hall, 1988) 79.

12. Peter Newmark, 80. 
encompasses all knowledge, understandings, values, attitudes, feelings and beliefs transmitted in traditional forms, by word of mouth or by customary examples"13, se puede suponer que el lector meta debería sentirse identificado con la temática de entorno de los fragmentos.

La poesía está tradicionalmente compuesta por ritmo, y en el caso de los fragmentos insertos, por rima, una combinación musical exclusiva del género poético. Esta forma inspira una lectura pausada, cuidadosa, independiente y exclusiva, en espera de que el lector logre identificar - y para los propósitos de este análisis - identificarse con aquellos elementos alineados en sus versos: "The very appearence of the poem on the page, its vertical arrangement of language in parallel lines, suggests that it needs to be read in a particular way" ". Es cierto que la transposición en prosa de lo escrito en verso puede aportarle al lector una idea general del contenido denotativo del poema. Sin embargo, "el impacto [del verso] se alcanza casi exclusivamente a través de las estrechas semejanzas en rima y ritmo" "15. Históricamente, el traductor siempre ha tenido problemas al enfrentar la traducción poética, no sólo por la presencia del contexto cultural, sino por la musicalidad de su forma. Según Widdowson "16: "We cannot simply convert the meaning of a poem into something more manageable, something more accord with convention, by producing a prose paraphrase or statement of gist. For to do this is to change the poem's pattern, deprive it of its peculiarity and therefore of its point". Pese a esto, algunos afirman que el proceso de lograr una traducción poética fiel y que, al mismo tiempo, mantenga los patrones de rima y ritmo del original, es una empresa demasiado laboriosa y fácilmente reemplazable con otros recursos traductológicos válidos como la simple paráfrasis. El presente tratamiento se orienta a un análisis mucho

13. Jean Harold Brunvand, The Study of American Folklore (Nueva York: W.W. Norton \& Company, 1978) 15.

14. Widdowson, 35.

15. Hatim y' Mason, 25.

16. Widdowson, 13. 
más completo y profundo como el que Paul Miclau ${ }^{17}$ defiende: "Si le traducteur se limite à la structure superficielle de l'original, qu'il transforme mécaniquement en structure superficielle dans la langue cible, alors il risque de realiser une traduction... superficielle". Lograr reproducir en el lector meta una sensación apelativa a los sabores de una cocina no se puede lograr si no se le transporta a un contexto poético semejante en que los "sabores" aparezcan en forma natural. Como afirma Widdowson ${ }^{18}$ :

Many readers will, of course, resist the suggestion, be disinclined to make the effort, and will not want to go beyond what can be transposed into conventional terms. Others will be prepared to be drawn into the poem and be ready to adapt to its demand, attracted perhaps by a certain cadence or particular image, intrigued to know how these might contribute to some underlying coherence made out of meanings along other dimensions beyond those which are immediately apparent.

El traductor debe evitar los métodos simplistas para traducir poesía producto del "miedo reverencial al género" 19 . Reproduciremos no sólo el sentido del T.O. sino además su estilo, la rima festiva, el tono sencillo y desenvuelto y el ritmo deliberadamente reiterativo, elementos que en este caso resultan tan importantes como el propio contenido denotativo. Una traducción sin estos elementos no dará cuenta del espíritu de los versos. Se utiliza "not a silly one-to-one translation form" 20 , sino una forma que transporta la naturalidad del discurso poético y que permite adaptarse a sus demandas de forma.

17. Paul Miclau, "La traduction de l'image poétique". Retour à la traduction (Paris: La Français Dans le Monde, 1987) 69.

18. Widdowson, 15.

19. Schökel y Zurro, 404.

20. Newmark, 13. 
Una vez definido el subgénero, se deben identificar aquellos elementos folclóricos presentes en el T.O. conocidos por el lector de la versión traducida, que le provoquen el deseo de revivir su propio pasado lleno del sabor de la cocina de antaño, tal como le habría sucedido al lector original. Brunvand ${ }^{21}$ relaciona el contenido de las tradiciones subyacentes en el texto folclórico con su forma y estilo propios: "Folklore comprises the unrecorded traditions of people; it includes both the form and the contents of these traditions and their style or technique of communication from person to person". Además, presenta cinco características del texto folclórico, fácilmente reconocibles en los fragmentos poéticos que nos ocupan: "its content is oral (usually verbal), or custom-related or material; it is traditional in form and transmission; it exists in different versions; it is usually anonymous; it tends to become formularized, that is expressed partly in clichés"22. El texto folclórico oral, aparte de revelar aspectos importantes acerca de la conducta cultural, también va a fomentar la creación de cambios, ligados con su naturaleza misma: "Oral transmission invariaby creates different versions of the same text, and these variations or variants are the third defining characteristic of folklore. [An oral text] is living folklore as long as it continues to circulate orally in different traditional variations" 23 .

Siendo el folclor agente de cambio, "no se puede reconstruir la situación cultural en la que nació el escrito, aunque sí es posible que el traductor elija el sentido que parece mejor avalado y lo refle je en el texto"24. Este es el valor de la conducta cultural, que en palabras de José Ortega y Gasset ${ }^{25}$, sería “entrever lo que traducir puede tener de magnífica empresa: la revelación de secretos mutuos que pueblos

21. Brunvand, 2 .

22. Brunvand, 12.

23. Brunvand, 16.

24. Nida, 24.

25. José Ortega y Gasset, "Miseria y esplendor de la traducción”. Miguel Ángel Vega, ed. Textos clásicos de teoría de la traducción (Madrid: Ediciones Cátedra, 1994) 301-302. 
y épocas se guardan recíprocamente y tanto contribuyen a su dispersión". Ahora bien, se ha creído necesario evaluar la conveniencia de todas y cada una de las referencias culturales presentes en los fragmentos. Se han encontrado estrechas relaciones históricas y culturales entre los contextos original y terminal, que han guiado la toma de decisiones en la búsqueda de las correspondencias léxicas y las transformaciones necesarias: "Translators need to understand the position of the source text in the source culture; without such knowledge they cannot cast around for the relevant analogies in the target literature and target culture. They may want to think of acceptable analogies that might serve as substitutes" 26 .

Se procura, entonces, la creación de un balance entre los elementos que se deben rescatar, "sin que esto produzca ninguna modificación esencial en la función de la poética"27. Miguel Ángel Vega ${ }^{28}$ indica cuales son estos aspectos: "El traductor deberá tener en cuenta las diferencias de estratificación social, de estilo de vida y las diferencias de cada cultura y subcultura ya que de todas estas condiciones dependerá un transvase cultural completo". Sin embargo, es Margaret Sayers Peden ${ }^{29}$ quien aporta una respuesta completa: "We can not translate until we "do violence" to the original literary work; we must de-struct it. Now the translator must sift through the rubble of his/her de-struction and rescue such materials as meter, rhyme, vocabulary, rhetorical tone, poetic figure and period".

La búsqueda de una reorganización que capture la naturalidad del sentido nos lleva a preguntar cuáles elementos se deben rescatar en el proceso de reconstrucción de los fragmentos. Newmark ${ }^{30}$ comienza a orientarnos al respecto: "the word order is the most dedicate indicator of naturalness". Schökel y Zurro ${ }^{31}$ también hacen mención a

26. Susan Bassnett-McGuire, Translation Studies (Londres: Routledge, 1999) 92.

27. Octavio Paz, "El soneto en Ix". El signo y el garabato (México: Mortiz, 1973) 78.

28. Miguel Ángel Vega, ed. La palabra vertida: investigaciones en torno a la traducción (Madrid: Ed. Complutense, 1997) 503.

29. Margaret Sayers Peden, "Building a Translation, the Reconstruction Business: 'Poem 145' of Sor Juana Ines de la Cruz". The Craft of Translation (Chicago: University of Chicago Press, 1989) 23.

30. Newmark, 49.

31. Schökel y Zurro, 120. 
este recurso cuando afirman que gran parte de los proverbios bíblicos se pueden traducir en formas castellanas rimadas si se dispone del orden de palabras y a la vez de los sinónimos. Ahora bien, al considerar los sinónimos, se debe tomar en cuenta que los equivalentes de las palabras que riman en inglés, por lo general, no conservan esa rima en español. Dado que el valor del significado predomina sobre la forma, muchos traductores han buscado la fidelidad al contenido del texto poético prescindiendo de la forma rítmica o de la forma métrica. Para los teóricos, esos recursos pueden ser válidos si se mantiene una calidad literaria aceptable en el producto final. Sin embargo, hay un elemento del cual no podemos prescindir: el ritmo, porque este es el elemento formal de más peso en el género poético. Para reproducir una poesía escrita en inglés, es necesario crear otra poesía escrita en español que conserve el sentido global del texto, pero que no parezca forzada. La construcción de un auténtico poema requiere de la valoración de su estética.

Umberto $\mathrm{Eco}^{32}$ define la "equivalencia funcional" de la siguiente manera: "...a good translation must generate the same effect aimed at by the original. Obviously this means the translators have to make an interpretative hypothesis about the effect programmed by the original text". Esteban de la Torre ${ }^{33}$, por su parte, afirma que "la forma que la expresión adquiere en el texto poético es de una relevancia crucial. Lo verdaderamente importante es crear —o recrear - un poema, un auténtico poema, escrito con versos frescos...". Octavio $\mathrm{Paz}^{34}$, en el análisis que él mismo le efectúa a su propia traducción del poema "El soneto en Ix" de Mallarmé, afirma que, al comparar el poema en castellano con el original, el suyo es "tal vez más libre pero con rimas de dificultad y sonoridad análogas". La sonoridad análoga que se pretende lograr toma en cuenta los efectos sonoros de la rima asonante por ser parte de los factores de los que dispone el género. Según Schökel y Zurro:

32. Umberto Eco, Experiences in Translation (Toronto: University of Toronto Press, 1998) 45.

33. Esteban de la Torre, Teoría de la traducción literaria (Madrid: Síntesis, 1994) 207.

34. Paz, 90. 
La rima consonante fácilmente dará la impresión de pobre, ingenua o demasiado rebuscada, mientras que la rima asonante es mucho más ligera y fácil, ayudará a la memoria y no dará la impresión de rebuscada. No es raro que en una serie silábica se cometan infracciones justificadas por el principio acentual. Esta es la situación frecuente, en nuestros cancioneros, hasta convertirse esa flexibilidad rítmica en una de sus características ${ }^{35}$.

Esteban de la Torre ${ }^{36}$ afirma que: "El texto español dispone de más sílabas, más palabras, más posibilidades expresivas... la traducción del verso ha de basarse, por lo tanto, y necesariamente, en el concepto de equivalencia rítmica”. Schökel y Zurro ${ }^{37}$, por su parte, amplían: "El ritmo acentual se puede emplear contando con rigor un número regular de acentos en los versos, aún cambiando su valor silábico; también se presta a combinaciones libres de número de acentos en las que el oído dictará sentencia. Acertar sin el apoyo de un esquema fijo será más difícil".

La presencia de rima supone una complicación adicional a la labor del traductor. Sin embargo, este proceso de traducción de los versos insertos ha mostrado que, a veces, basta con sólo ajustar la rima del verso inicial para que la traducción sea estéticamente acertada, y escoger un lexema análogo para lograr una versión en español con correspondencia analítica y sonoridad análoga. La rima es un uso particular y extraño en la lengua que se lleva a cabo en forma consciente y sistemática. De ese mismo modo, su traducción ha de ser consciente y sistemática, y en función del rescate de sus elementos métricos, de rima y de ritmo presentes con una intencionalidad puramente emotiva.

Para realizar la traducción de textos de tema religioso, se requiere tomar en cuenta la naturaleza universal de su contenido. Debido a

35. Schökel y Zurro, 150-151.

36. De la Torre, 207.

37. Schökel y Zurro, 153. 
que los hebreos componían toda su poesía religiosa para ser leída, declamada o cantada, el factor sonoro desempeña un papel muy importante para la creación de un ambiente solemne ${ }^{38}$. Las peculiaridades de cada uno de los fragmentos insertos han exigido un tratamiento distinto en lo relativo a la métrica y al rescate del mensaje y de su función dentro de la prosa. Hemos de recordar que la cantidad de palabras de un texto escrito originalmente en inglés aumenta como mínimo en un diez por ciento con respecto a su equivalente traducido en español. La pérdida, entonces, de algunos lexemas se justifica ante la sencilla razón de que éstos no cuentan con suficiente espacio material en la lengua meta. El lector de la traducción encontrará un verso ágil y con efecto sonoro, con un orden de los lexemas que ha variado ligeramente.

Sobre el valor de la musicalidad poética en la traducción, Esteban de la Torre ${ }^{39}$ señala lo siguiente: "El carácter melodístico de la expresión poética no es algo secundario, añadido o superfluo sino, por el contrario, un elemento imprescindible, esencial, que hay que tomar en cuenta si se pretende que la versión de un poema sea un poema". Ahora bien, debido a que, en el castellano, el hablante cuenta sílabas, mientras que en inglés cuenta acentos fonéticos, resulta inevitable efectuar cambios en el orden de los elementos, como lo apunta Newmark, con el fin de rescatar la musicalidad poética. Para Schökel y Zurro ${ }^{40}$, esta musicalidad no debe ser impuesta, sino natural:

La traducción española no puede reproducir cada efecto sonoro, ni hace falta... el efecto no reside en cada detalle, sino más bien en el clima total... Se trata más bien de entrar en un ámbito sonoro escuchando el original y escuchando nuestras canciones; después de los tanteos, hallazgos y correcciones, el oído estará siempre vigilante para aceptar o rechazar.

38. Schökel y Zurro, 168.

39. De la Torre, 199.

40. Schökel y Zurro, 160. 
Como afirma De la Torre ${ }^{41}$ :

...lo que al traductor realmente le interesa es localizar en el TLO [texto en la lengua original], las estructuras sonoras que sean estéticamente pertinentes que añadan algún matiz semántico o estético a los significados puramente denotativos. El traductor deberá, por lo tanto, advertir el recurso fonoestilístico que se utiliza y después tratar de reproducirlo en el TLT [texto en la lengua terminal] mediante algunos de los procedimientos.

Según Eugene Nida ${ }^{42}$, al traducir textos bíblicos o textos provenientes de la práctica cristiana, hay que tener en cuenta que "los autores de los libros bíblicos querían hacerse entender; ...por lo que no hay que traducirlos como si fueran cantos rituales ugáricos, sino como himnos utilizados en el culto de Yahvé". Peter France ${ }^{43}$ describe la propuesta de Nida: "it uses language which is natural, clear, simple, and unambiguous, following the principle of dynamic equivalence that results in a vigorous and uncluttered style, which has been particularly welcomed among younger people for whom Bible English is an unfamiliar language". Todas las consideraciones teóricas anteriormente expuestas fundamentarán, entonces, la estrategia de traducción a seguir. En 1533, Martín Lutero ${ }^{44}$ escribió:

...quizás habrá algunos en nuestro tiempo y muchos en el venidero que pudieran escandalizarse porque nosotros hayamos procedido en muchos pasajes apartándonos de la letra y a veces entendiendo un sentido distinto del que captan los rabinos y los gramáticos judíos; queremos aquí mostrar los motivos y explicar

\section{De la Torre, 164 .}

42. Nida, 23.

4.3. Petcr France, The Oxford Guide to Literature in English Translation (Nueva York: Oxford University Press, 2000) 165.

44. Martín Lutero, "Sumarios sobre los salmos y motivos de la traducción”. Textos clásicos de teoría de la traducción (Madrid: Ediciones Cátedra, 1994) 112. 
con algunos ejemplos en los que vean como nosotros no hemos procedido así por falta de entendimiento de la lengua ni por ignorancia de las glosas de los rabinos, sino que nos hemos propuesto semejante traducción a ciencia y conciencia.

Sin duda, esta empresa es un desafío: no puede ser lineal porque se alejaría de la auténtica traducción literaria; por el contrario; debe ser persuasiva para que permita evocar ese sabor regional, al mismo tiempo que vela por la naturalidad de una forma poética transpuesta en otra lengua.

\section{El tratamiento completo}

Llamaremos el tratamiento completo a la metodología propuesta para facilitarle al lector terminal el acceso al contenido y a todos los elementos semánticos e interpretativos que corresponden a los fragmentos poéticos. Este procedimiento no se limita simplemente a ejemplificar las consideraciones teóricas expuestas, sino a sistematizarlas "sintonizando el tono del texto original" manifiesta que el propósito del libro es "demostrarle al lector que la vida hogareña cumple un papel importante en nuestra historia"46. Una nueva lectura de los fragmentos permitió establecer una relación entre las vivencias allí descritas y las propias; precisamente por su relevancia "en nuestra historia". La traducción de los fragmentos busca rescatar lo autóctono, lo esencial y lo propio del entorno del lector terminal, ya que al carecer los fragmentos de un final o por encontrarse segmentados, la traducción "debe completarlos" haciendo uso de elementos afines al contexto al que va dirigida la traducción.

La naturaleza misma de esta motivación permite comprender la función de los fragmentos insertos que conlleva una visión

45. Schökel y Zurro, 156.

46. Tartan, 6 . 
reminiscente, producto de las semejanzas contextuales entre el entorno de cada fragmento y la experiencia del lector terminal. La presencia de un breve fragmento poético, sin ninguna especificación sobre su origen ni sobre su contenido, resulta suficiente para el lector del T.O., debido a que él probablemente conocerá su procedencia. Sin embargo, el lector terminal requiere que se le transporte más allá de esa estructura superficial a razón de establecer toda la asociación idiosincrática que ha guiado la etapa final de la traducción. La reacción sentimental está extrechamente ligada con las motivaciones ocultas en los fragmentos de poesía; se va más allá de la interpretación convencional hasta el punto de aparecer "drawn into de poem" 47 , entendiendo así a una nueva dimensión del sentido.

El tratamiento completo, el esquema fijo, que Schökel y Zurro recomiendan seguir, agrupa una parte de la gran cantidad de consideraciones teóricas existentes con respecto a la traducción de poesía, folclor y cultura sistematizadas en doce pasos aplicables a todos los fragmentos poéticos insertos:

\section{La identificación del poema de procedencia}

$\mathrm{El}$ poema de procedencia, que también llamaremos, poema fuente o poema de origen, es el texto completo de donde proviene el fragmento inserto. La información sobre los poemas de origen se obtuvo investigando distintos textos paralelos, entrevistando a hablantes nativos del inglés y por medio de la Internet.

\section{La relación del fragmento con el resto del poema}

Es necesario observar con cuidado la posición que tiene el fragmento inserto en el T.O. dentro del poema fuente. Se ha comprobado que la estructura de los fragmentos, así como sus diferentes características de ubicación conllevan a distintas consideraciones en el momento de traducirlos.

47. Widdowson, 15 . 
3. Corroborar los significados de cada una de las palabras

Por tratarse de un contexto regional folclórico, es necesario revisar el significado de cada uno de los lexemas no sólo haciendo uso del diccionario especializado, sino de suficientes textos paralelos.

\section{La traducción lineal de los versos}

Resulta necesario traducir los versos en forma literal o lineal, con el fin de verificar la presencia y posición de todos los elementos léxicos y sintácticos del poema e iniciar la visualización de su relación sintáctico-semántica.

5. Determinar la carga semántica de las palabras y su jerarquía Se clasificarán los lexemas del poema completo en lexemas de primer, segundo y tercer orden ${ }^{48}$. Esta primera categoría de lexemas es la de rigor, que deberá estar presente en la versión traducida del poema. Los lexemas de segundo orden son términos o elementos textuales, también de gran riqueza semántica, que el traductor podrá utilizar en forma complementaria para ayudarse en el rescate del ritmo y la rima. Con respecto a los lexemas de tercer orden, el traductor podrá manipularlos con mucha más facilidad, al punto que puede prescindir de sus equivalentes en la lengua meta.

\section{Establecer la relación sintáctica entre los versos}

Es necesario analizar en detalle cuáles versos están sintácticamente relacionados entre sí para efectos de intentar trasladar esa relación a la lengua meta.

7. Buscar varios sinónimos de los lexemas de primer orden

Se recurre a este paso si el equivalente más próximo en castellano no facilita la labor de mantener la rima o el ritmo o si resulta del todo inadecuado para los propósitos de la traducción. Una vez efectuada

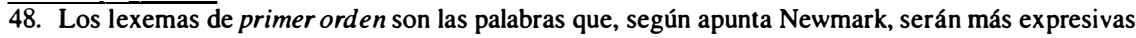
debido a su carga semántica. 
esta búsqueda, se procede a seleccionar los sinónimos que por su acentuación y número de sílabas contribuyen a mantener el ritmo. En este paso se procede también a ubicar los lexemas que mejor correspondan a los elementos de tipo fonológico (por ejemplo, interjecciones) o exclusivos del folclor presentes en el poema de origen.

8. Reevaluar la selección de términos con el propósito de mantener la rima

Atendiendo a la indicación de los teóricos en relación con el rescate de la espontaneidad y la conveniencia de la rima asonante, reevaluaremos el uso de los lexemas de primer y segundo orden, así como sus respectivos sinónimos, en función del rescate de la rima. Es imprescindible tomar en cuenta en este paso, que lo más importante para los propósitos de nuestra traducción es procurar el rescate de las formas rítmica y rimada de los poemas que contribuyen a la transmisión de la función apelativa.

9. Contar las sílabas de los versos traducidos

Debemos corroborar la correspondencia del número de sílabas con el ritmo del poema completo para mantener su musicalidad. Para la traducción de versos musicales (por ejemplo, villancicos) debemos contabilizar con sumo cuidado el número de golpes y acentos rítmicos para que correspondan con la música de la partitura.

\section{Velar por la correspondencia del ritmo}

Se vuelve al poema original, que momentáneamente se ha dejado de lado, y se efectúa un acercamiento contrastivo entre el ritmo, los acentos y las características estructurales de ambas versiones; este paso es necesario, con el fin de asegurar la correspondencia funcional que se desea que ambos poemas mantengan entre sí. 


\section{Realizar cambios de orden sintáctico y terminológico}

Será necesario ef ectuar cambios una vez que se "ha concluido" la traducción de los poemas. Resulta conveniente hacer notar que entre los pasos 10 y 11 se ha de dejar un intervalo temporal suficiente, con el fin de lograr una mejor visualización de los resultados.

\section{Insertar el fragmento poético en el T.T.}

Concluido este proceso de traducción y edición, procederemos a analizar cuidadosamente la forma que ha tomado el nuevo fragmento poético y las justificaciones que apoyen su reaparición en el T.T.

Cabe preguntarse por el destino del resto de los poemas originales, también traducidos como producto de la aplicación del tratamiento completo, una vez inserta la nueva versión del fragmento en el T.T. Los poemas traducidos $y$ las consideraciones referentes al trabajo realizado deberían incluirse como parte de la publicación, en un prólogo, epílogo, apéndice o por otro recurso similar, tal y como lo hacen reconocidos autores y traductores, como Paz y Eco, con el fin de exponer los alcances de su trabajo.

Beth Tartan escribió su libro para que sus coterráneos saborearan de nuevo todas esas delicias que, como ya vimos, van mucho más allá de un plato de sopa o de una taza de café. Fuera de su mundo, es necesario que nosotros, los lectores costarricenses, conozcamos la totalidad del poema fuente. Sólo de esta forma lograremos saborear las notas de su música, el ritmo alegre de su rima y adentrarnos en el alma de una niña que, a su manera, quiso transportarnos a sus raíces. Un tratamiento absolutamente literal hubiera privado a la traducción de su libertad expresiva y de todo el sabor que la autora deseaba transmitirnos. La traducción del verso en forma literal o en forma de prosa, a lo mejor válida para otro tipo de poemas, hubiera apartado al lector terminal de unos poemas que no se insertaron al azar. De haber sido indiferente a la percepción estética de estos fragmentos, no se habría logrado que el texto traducido pueda, acaso, ser percibido como 
si originalmente hubiera sido escrito en esa lengua. Por supuesto que se pudo haber prescindido de la rima; sin embargo, esto no hubiera transvasado adecuadamente la sugestividad acústica del poema original. Es necesario guardar una estricta equivalencia semántica con las pautas del poema de origen, aunque esto implique reacomodar su contenido con respecto a su estructura y su significado.

$\mathrm{El}$ acto de la traducción es un proceso comunicativo en el que la elección onomasiológica del autor del T.O. debe ser interpretada en forma semasiológica dentro de la propia lengua del traductor. Esto es lo que permite producir un nuevo texto, equivalente en sentido, función y sabor al original. Si el traductor se limita a traducir los significantes, la forma de expresión carece, entonces, de interés y aleja al lector de la traducción de la posibilidad de disfrutar un texto con una estructura literaria ajena a la realidad poética del T.O. En la traducción de un texto poético escrito en verso, la forma de expresión está unida al contenido, por eso es que ésta adquiere relevancia en la recreación del poema en otra lengua. Lo verdaderamente importante es recrear este poema a través de cambios que revelen la estructura poética esencial, pero con una simplicidad natural que evoque en el lector el sentido de las palabras mismas.

Al replegar el movimiento del significado interior y los momentos simbólicos que ocurren en cada poema, los signos musicales enlazan aún más esa relación entre el significante y el significado; relación que, de omitirse, conlleva a la pérdida de algo esencial: el mensaje del poeta y de la realidad que el poema encierra. Esta realidad es un signo más que hay que traducir como parte del momento natural de la idea hecha signo. Se debe vislumbrar la relación que une el significante con el significado de una poesía a través de los distintos recursos de traducción; tomando conciencia de la relación de los signos del texto en su lengua.

La fidelidad al T.O. nunca se ha perdido de vista; sin embargo, en la medida de lo posible, tampoco se ha prescindido de los elementos de métrica, rima y ritmo. La traducción de estos fragmentos insertos, 
lejos de ser un problema más bien se ha convertido en una empresa sumamente placentera en la que, por todos los medios, se ha evitado la labor mecánica de trasladar palabra por palabra lo que dicen los significantes, con el fin de que el lector no perciba los poemas como traducciones de una lengua extranjera, sino como poesías escritas en castellano capaces de transportarlo a sus vivencias religiosas, folclóricas y culturales.

Las características melódicas le dan una extraordinaria riqueza semántica al poema; se constituye en todo un reto lograr entrelazar los significantes de manera que alcancen un ef ecto emotivo en el lector de la traducción. Hay cambios formales evidentes que están en función de las necesidades métricas y de las peculiaridades de la lengua a la que se traduce. Se intentó reproducir los lexemas expresivos, así como las ideas fundamentales. En lo referente a la sonoridad, se hizo el mejor esfuerzo para mantenerla, debido a que ésta es el soporte semántico que alterna el simbolismo fónico con la motivación hacia la función del fragmento del poema. Los significados presentan un entramado acústico de dependencia interna entre los fonemas y los lexemas, entre el significante y el significado, entre las palabras y el sentido. La traducción de un poema no puede limitarse a traducir el esqueleto del sentido, sino que debe tener muy en cuenta todo aquello que está vivo y que palpita bajo la piel de la palabra.

Este ejercicio, aparte de ser un acto genuino de respeto a la función del verso inserto en la prosa no literaria, se manifiesta en sí mismo como una actividad creadora. En vez de ir en contra del cuerpo del poema, se utiliza la creación de una palabra bella. Es cierto, se experimentan muchas restricciones, pero al final se aprende a lidiar con ellas. Placentero resulta haber logrado plasmar el sabor de las vivencias que se describen en el T.O. Estos poemas son sentimientos hechos palabras, un momento plasmado en el tiempo. En North $\mathrm{Ca}$ rolina and Old Salem Cookery, los significantes asumbran con su presencia, maravillan a quien los recibe y poseen un sabor tan profundo como el de su significado. 